\title{
BMJ Open Clinical and metabolic characteristics of the Diabetes Intervention Accentuating Diet and Enhancing Metabolism (DIADEM-I) randomised clinical trial cohort
}

Hadeel Zaghloul, ${ }^{1,2}$ Odette Chagoury, ${ }^{1,3,4}$ Sara Elhadad, ${ }^{1}$ Salma Hayder Ahmed, ${ }^{1}$ Noor Suleiman, ${ }^{1,2,3,4}$ Abdulla Al Naama, ${ }^{5}$ Katie El Nahas, ${ }^{6}$ Abdulla Al Hamaq, ${ }^{6}$ Mary Charlson, ${ }^{2}$ Martin T Wells, ${ }^{7}$ Samya Al Abdulla, ${ }^{5}$ Abdul Badi Abou-Samra,,${ }^{1,2,3}$ Shahrad Taheri (D) 1,2,3,4

To cite: Zaghloul $\mathrm{H}$ Chagoury 0, Elhadad S, et al. Clinical and metabolic characteristics of the Diabetes Intervention Accentuating Diet and Enhancing Metabolism (DIADEM-I) randomised clinical trial cohort. BMJ Open 2020;10:e041386. doi:10.1136/ bmjopen-2020-041386

- Prepublication history and additional material for this paper is available online. To view these files, please visit the journal online (http://dx.doi.org/10. 1136/bmjopen-2020-041386).

Received 09 June 2020 Revised 29 0ctober 2020 Accepted 19 November 2020

Check for updates

(c) Author(s) (or their employer(s)) 2020. Re-use permitted under CC BY-NC. No commercial re-use. See rights and permissions. Published by BMJ.

For numbered affiliations see end of article.

Correspondence to Professor Shahrad Taheri; staheri@me.com

\section{ABSTRACT}

Objectives Diabetes Intervention Accentuating Diet and Enhancing Metabolism-I (DIADEM-I) is the first randomised controlled trial (RCT) in the Middle East and North Africa (MENA) region testing the effectiveness of an intensive lifestyle intervention (ILI) for weight loss and diabetes remission. We report on the recruitment process and baseline characteristics of the DIADEM-I cohort based on origin (Middle East vs North Africa), and waist circumference.

Design DIADEM-I is an open-label randomised, controlled, parallel group RCT recruiting young individuals (18-50years) with early type 2 diabetes ( $\leq 3$ years since diagnosis) originating from MENA. Individuals from primary care were randomised to usual medical care or ILI (total dietary replacement phase using meal replacement products, followed by staged food reintroduction and physical activity support). The primary outcome is weight loss at 12 months. Other outcomes are glycaemic control and diabetes remission.

Setting Primary care, Qatar.

Participants 147 (73\% men) randomised within DIADEM-I who were included in the final trial data analysis Outcome measures Recruitment metrics, and baseline clinical and metabolic characteristics.

Results Of 1498 people prescreened, 267 (18\%) were invited for screening and 209 (78\%) consented. 173 (83\%) were eligible. $15(7 \%)$ withdrew before randomisation and the remaining 158 were randomised. Mean age was 42.1 (SD 5.6) years and mean body mass index was: 36.3 (5.5) $\mathrm{kg} / \mathrm{m}^{2}$ (women) and $34.4(5.4) \mathrm{kg} / \mathrm{m}^{2}$ (men). Mean diabetes duration was 1.8 (1.0) years and mean glycosylated haemoglobin ( $\mathrm{HbA1c}$ ) was $7.0 \%$ (1.30) $(52.5 \mathrm{mmol} / \mathrm{mol}$ (SD 14.3)). Participants originated from 13 countries. Those from North Africa reported greater physical activity and had lower family history of diabetes. $90 \%$ of subjects were taking diabetes medications and $31 \%$ antihypertensives. Those with greater waist circumference had significantly higher insulin resistance and lower quality of life.

Conclusion Recruitment of participants originating from the MENA region into the RCT was successful, and
Strengths and limitations of this study

- This work describes key clinical and metabolic characteristics of participants in Diabetes Intervention Accentuating Diet and Enhancing Metabolism-I (DIADEM-I), the first randomised controlled trial of an intensive lifestyle intervention for weight loss and type 2 diabetes (T2DM) remission in the Middle East and North Africa (MENA).

- Recruitment of participants originating from 13 different countries into the study was successful from primary care in Qatar and included a high percentage of men.

- Findings from the DIADEM-I study will provide key evidence for the management of T2DM in MENA and globally.

- The characteristics described for those with early T2DM in MENA are limited to a single study.

study participation was readily accepted. While DIADEM-I participants originated from 13 countries, there were few baseline differences amongst participants from Middle East versus North Africa, supporting generalisability of RCT results.

Trial registration number ISRCTN20754766; NCT03225339

\section{INTRODUCTION}

The prevalence of type 2 diabetes (T2DM) is increasing globally in parallel with the increasing prevalence of obesity. The Middle East and North Africa (MENA) region has a high prevalence of obesity and in 2019 had the highest age-adjusted diabetes prevalence for adults (20-79 years) worldwide of $12.2 \%$ with a projected prevalence of $13.3 \%$ and $13.9 \%$ for 2030 and 2045, respectively. ${ }^{1}$ It is estimated that by 2045 the number of 
people with diabetes in MENA will increase by $96 \%$, the second highest increase worldwide. ${ }^{1}$ The main drivers for the rise in diabetes prevalence in MENA are rapid development and urbanisation, which have promoted an increased intake of energy dense foods and sedentary lifestyles. Diabetes in MENA occurs at a younger age group than white European populations resulting in greater morbidity and early mortality. In 2019, diabetes and its complications caused $16.2 \%$ of all deaths in the MENA region and $22.4 \%$ of these deaths were in the $30-39$ years age group. ${ }^{1}$ More than half the deaths were in those under 60 years of age. ${ }^{1}$

The prevailing approach to diabetes management has been to focus on glycaemic, lipid and blood pressure control through medication introduction and escalation, ${ }^{2}$ based on the assumption that T2DM is a life-long disease with progressive decline in beta cell function and greater atherosclerosis. Increasingly, this view has been questioned through observations from bariatric surgery ${ }^{34}$ as well as recent dietary intervention trials, ${ }^{5-7}$ where diabetes remission was observed to accompany significant weight loss. Excess adiposity is a key driver for T2DM and significant weight loss, either through bariatric surgery or medical management, can improve and potentially reverse diabetes. The cost and limited availability of bariatric surgery, however, make it an unrealistic solution for the large population with T2DM and obesity. ${ }^{8}$ Most recently, the Diabetes Remission Clinical Trial (DiRECT) ${ }^{5}$ employing a total diet replacement (TDR) phase using low energy diet (LED) meal replacement products, examined the effect of weight loss through dietary intervention on achievement of T2DM remission. The study included 298 individuals with T2DM (mean age and diabetes duration of 54.4 and 3 years, respectively). Diabetes remission was reported in $46 \%$ of the intervention group at 1 year $^{5}$ and $35.6 \%$ at 2 years. ${ }^{6}$

While bariatric surgery is increasingly used for T2DM management in the MENA region, ${ }^{9}$ there have been no trials of intensive lifestyle intervention (ILI) for weight loss and T2DM remission in a region with a high prevalence of obesity and T2DM in younger individuals. The Diabetes Intervention Accentuating Diet and Enhancing Metabolism-I (DIADEM-I) was designed as the first randomised clinical trial in the MENA region to examine the impact of an ILI that included a TDR phase using LED meal replacements combined with physical activity on body weight, and glycaemic control and diabetes remission in those who are young (aged 18-50 years) with early T2DM ( $\leq 3$ years). ${ }^{10}{ }^{11}$ DIADEM-I hypothesised that intervention in young individuals in the early stages of T2DM through significant weight loss, behaviour change and physical activity would translate into a significant percentage reversing T2DM. Indeed, DIADEM-I recently reported a significant weight loss of about $12 \mathrm{~kg}$ in the intervention group at 12 months that was accompanied by a $61 \%$ remission of diabetes and $33 \%$ reversion to normoglycaemia. ${ }^{11}$ Here, we describe the baseline clinical and metabolic characteristics of participants in the
DIADEM-I study and provide insights into T2DM in those in the MENA region.

\section{METHODS}

\section{Study design}

DIADEM-I is an open-label, randomised controlled, parallel-group trial. The trial randomised individuals with early T2DM into two intervention arms on a 1:1 basis. The intervention arms included: (1) ILI incorporating a TDR phase using LED meal replacement products (Cambridge Weight Plan, Northants, UK) for 12 weeks followed by gradual food introduction (staged replacement of LED meal replacement products with participants' own meals) for a further 12 weeks and then 6 months follow-up on own diet. Follow-up is planned for a further 12 months. (2) Usual medical care consisting optimisation of diabetes, lipid-lowering, and blood pressure medications with medication selection aimed at optimising weight loss and its maintenance. Management was based on the American Diabetes Association (ADA) guidelines. ${ }^{12}$

The trial is registered with ISRCTN (ISRCTN20754766; date assigned: 7 June 2017), and ClinicalTrials.gov identifier (NCT03225339; registered on 26 June 2017). A detailed description of DIADEM-I has been published elsewhere. $^{1011}$

\section{Description of the ILI}

The lifestyle intervention is delivered by a single multiprofessional team (physicians, dietitians and physical activity trainers) in a primary care and community setting in Doha, Qatar. Behaviour support is provided throughout the intervention to aid weight loss and its maintenance.

\section{Diet}

Subjects receive dietary support through trained dieticians. The intervention is divided into five phases: Phase 1-12 weeks LED (seen biweekly); phase 2-12 weeks partial LED (seen biweekly); phase 3-6 months own food and lifestyle change (seen every 4 weeks); phase 4-12 months follow-up (seen quarterly); phase 5-poststudy follow-up (via medical records).

\section{Physical activity}

A key component is support for physical activity through trained physical trainers. Physical activity is prescribed by the trainer (visits simultaneous with dietician schedule) but carried out unsupervised by participants. Physical activity advice follows the American College of Sports Medicine and ADA joint position stand. ${ }^{13}$

\section{Outcome measures}

The clinical trial primary outcome is weight loss at 12 months. Key secondary outcomes are glycaemic control and diabetes remission. Diabetes remission in DIADEM-I is defined as $\mathrm{HbAlc}$ value of $<6.5 \%(<48 \mathrm{mmol} / \mathrm{mol})$ and receiving no pharmacological diabetes therapy for $\geq 3$ months. ${ }^{11}$ 


\section{Sample size calculation}

Sample size calculation was based on the primary outcome. The Look AHEAD (Action for Health in Diabetes) study estimated the standard deviation for weight loss across populations to be $9 \%$. Using analysis of covariance (ANCOVA), 69 subjects per arm would be needed to achieve the primary outcome after accounting for a $30 \%$ dropout rate, and to provide a power of 0.8 with 0.05 significance level. ${ }^{11}$

\section{Subject recruitment and randomisation}

Recruitment was between 16 July 2017 and 30 September 2018. The study recruitment benefitted from primary care electronic medical record system established across primary and secondary care in Qatar. The DIADEM-I research team collaborated with the primary healthcare management team. A search string to identify eligible subjects with T2DM was developed and a computerised search of primary care electronic medical records was run by primary care to identify patients who fulfil the inclusion criteria. A list was generated by the search and was screened by the clinical team in primary healthcare. Any individual who was determined to be unsuitable because of age, origin, body mass index (BMI) or duration of diabetes was removed from the list. Primary care nurses and physicians approached potential subjects and gauged interest in study participation. Interested individuals were then referred to the research team and invited to a consenting/screening visit. Written, informed consent was obtained by a trained member of the research team. All consented eligible subjects underwent baseline assessment and randomisation into the study arms. Allocation to study intervention arms was made by clinical research coordinators via a web-based system, which employed a pregenerated randomisation list.

\section{Eligibility criteria}

\section{Inclusion criteria}

Eligible subjects were those 18-50 years old with a reported diagnosis of T2DM in the previous 3 years, $\mathrm{BMI} \geq 27 \mathrm{~kg} / \mathrm{m}^{2}$, origin from MENA and resident in Qatar.

\section{Exclusion criteria}

Exclusion criteria were type 1 diabetes, ischaemic cardiovascular event in the previous 6 months, chronic kidney disease stage $3 \mathrm{~b}$ or greater, any condition precipitating fluid overload, significant diagnosed psychiatric disorder, uncontrolled depression, uncontrolled epilepsy, known lactose intolerance, severe arthritis preventing walking, active gout and active gallstone disease or known asymptomatic gallstones.

\section{Rationale for eligibility criteria}

Diabetes at a younger age is associated a longer disease burden and hence greater risk of macrovascular and microvascular complications. The objective of DIADEM-I was to recruit younger individuals than those commonly enrolled into diabetes and weight management studies. Therefore, the upper age limit was set at 50 years for several reasons. First, obesity and T2DM are increasingly affecting a younger age group with serious consequences for the affected individual, healthcare services and society. ${ }^{14}$ Second, data from bariatric surgery have shown that diabetes remission is most likely to occur in those who are younger, have early diabetes and are on lower number of medications. ${ }^{3}$ Finally, a key aim of the intervention was to encourage physical activity for weight loss maintenance and younger individuals are less likely to have any contraindications to physical activity. It was also for this reason that the study excluded those who had conditions such as arthritis or active cardiovascular disease (CVD) that would affect safe participation in more vigorous physical activity. A cut-point of 3 years was chosen for diabetes duration to ensure that participants were included at a stage where pancreatic beta cell dysfunction has the greatest chance of reversibility. Subjects with active gout were excluded in order to prevent disease exacerbation with weight loss. Active or asymptomatic gallstone disease was an exclusion criterion because rapid weight loss can be associated with formation of gallstones and exacerbation of gallstone disease. Women were also excluded if they were currently pregnant, lactating or planning pregnancy within the study period.

\section{Funding}

The study is funded by the Qatar National Research Fund through the National Priorities Research Program (NPRP) grant NPRP 8-912-3-192 (principal investigator: Taheri). There is no input from the sponsor or funding source into the design, conduct, analysis or reporting of results.

\section{Statistical analyses}

For this report baseline characteristics of participants whose data are included in the final analysis are analysed. Summary data are presented here for the baseline study population. Mean and SD are used to describe continuous variables and frequencies and percentages used for categorical data. The distributions of the continuous variables were assessed by visual inspection of histograms and normal probability plots. When the distribution of a variable was right skewed, a logarithmic transformation was applied and the distribution of the transformed data element was reassessed by visual inspection of the new histogram and normal probability plot. The two-sample Wilcoxon rank-sum test was used to analyse physical activity data. For comparison between groups, unpaired t-tests were used. Fisher's exact test was used for categorical data. A median split for waist circumference $(112.3 \mathrm{~cm})$ was performed to compare the metabolic characteristics within the cohort. Statistical analyses were done in Stata 15/MP. Summarised data for the study population are also compared with summary data of populations in other clinical trials for weight loss interventions in patients with diabetes in the USA and UK to compare study populations and for assessment of generalisability of the trial results. Baseline data from the intervention arms have been reported elsewhere with the primary outcome data. ${ }^{11}$ 


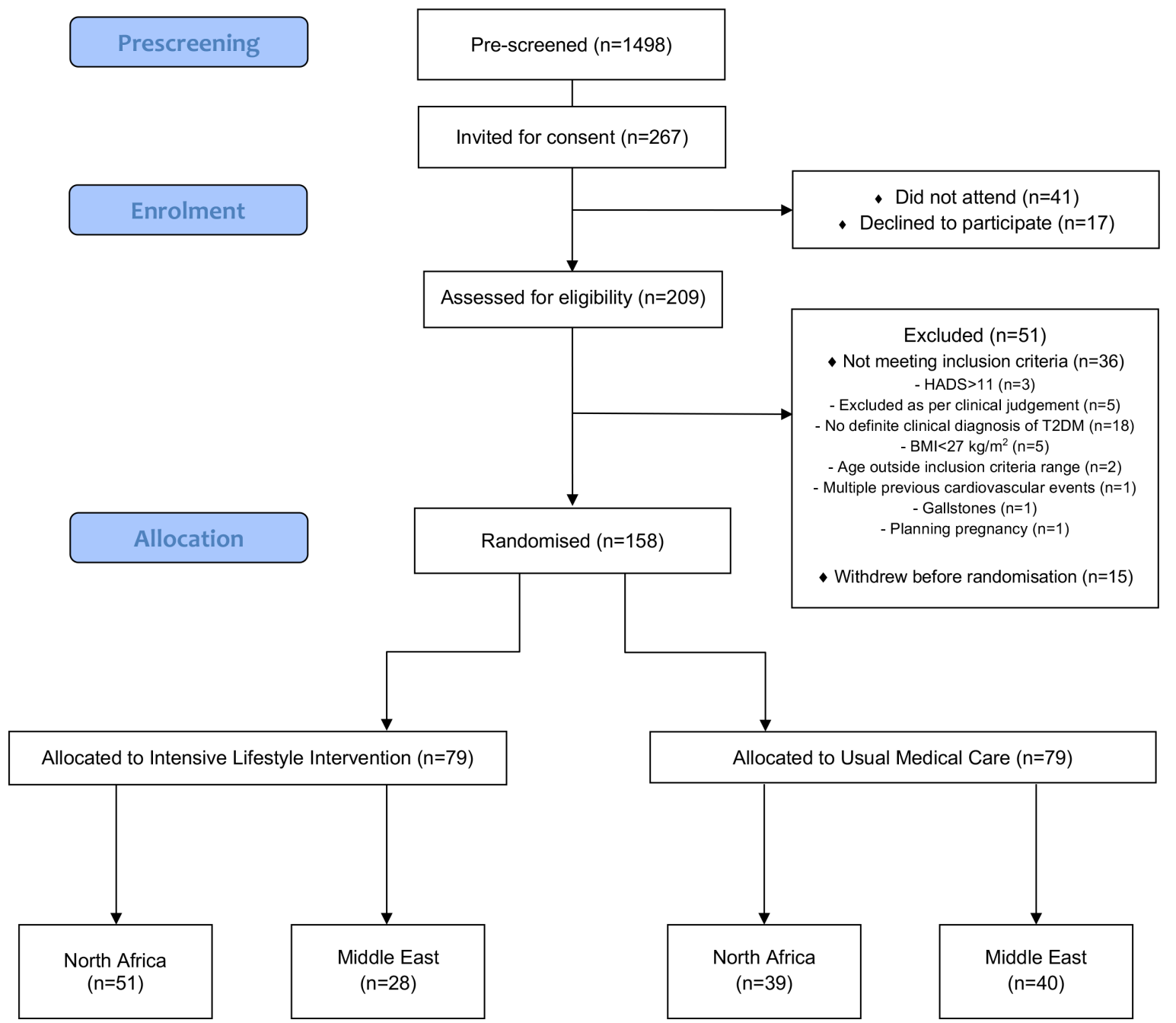

Figure 1 Flow chart of recruitment. BMI, body mass index; HADS, Hospital Anxiety and Depression Scale; T2DM, type 2 diabetes.

\section{Patient and public involvement}

Informal discussions with patients attending the weight management service at National Obesity Treatment Center, Qatar indicated the potential acceptability for the use of meal replacement products for management of obesity. Our previous work with the public suggested a willingness to participate in clinical trials. ${ }^{15}$

\section{RESULTS}

\section{Participant recruitment}

A summary of the recruitment process is found in figure 1 . Of the 1498 people prescreened for DIADEM-I by primary care, $267(18 \%)$ were invited for screening of whom 209 $(78 \%)$ accepted. Of the 209 consented participants, 173 $(83 \%)$ were eligible and $36(17 \%)$ were screen failures (figure 1). Fifteen consented subjects (7\%) withdrew before randomisation. Of those eligible, 158 participants were randomised into the trial $(\mathrm{N}=79 /$ study group). Three subjects were randomised in error (two because of diabetes duration $>3$ years, one due to significant $Q$ waves on baseline ECG). One subject was found to have type 1 diabetes after commencing the intervention. One was excluded on medical grounds because of severe asthma. Six subjects did not continue with the study or receive their baseline assessment. Data presented are for the 147 participants.

\section{Baseline characteristics}

Baseline characteristics of the participants are presented in tables 1 and 2. Summary data are presented for all participants and by region of origin (Middle East or North Africa). The mean age of the cohort at randomisation was 42.1 (SD 5.6) years. The study population was predominantly men $(73 \%)$, in line with the gender distribution of the population residing in Qatar, estimating the percentage of men in the population to be $76 \%{ }^{16}$ Participants originated from 13 different countries in the region. The mean BMI for participants was slightly higher in women than men (36.2 (SD 5.5) $\mathrm{kg} / \mathrm{m}^{2}$ and 34.4 (SD 5.4) $\mathrm{kg} / \mathrm{m}^{2}$, respectively). The mean duration of diabetes since diagnosis was 21.2 (SD 12.3) months. Mean HbAlc at baseline was 7.0\% (SD $1.30)(52.5 \mathrm{mmol} / \mathrm{mol}$ (SD 14.3)). At baseline, the majority of participants were taking more than three medications $(69 \%)$. Only $10 \%$ of participants managed their diabetes 
Table 1 Baseline characteristics by region of origin

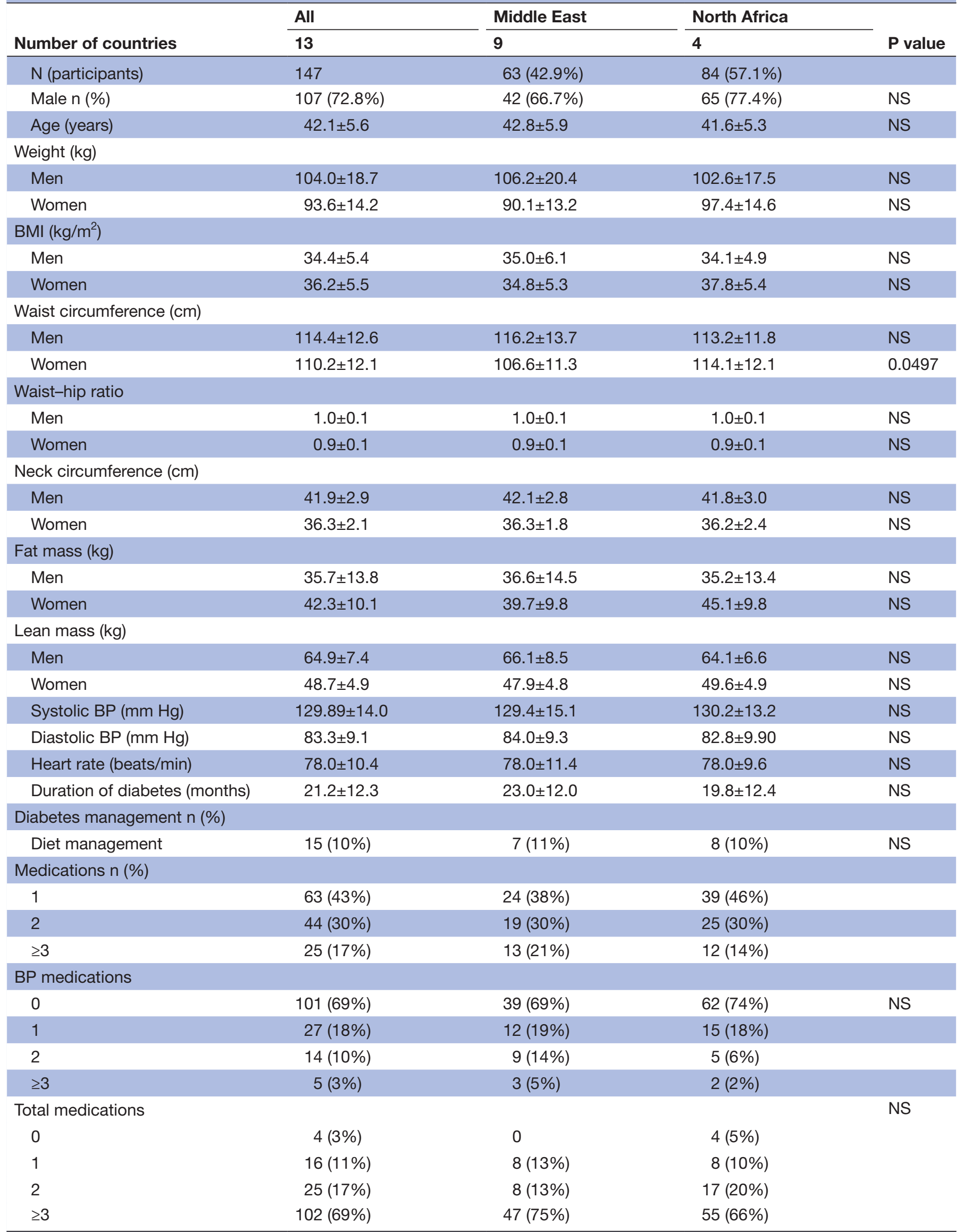


Table 1 Continued

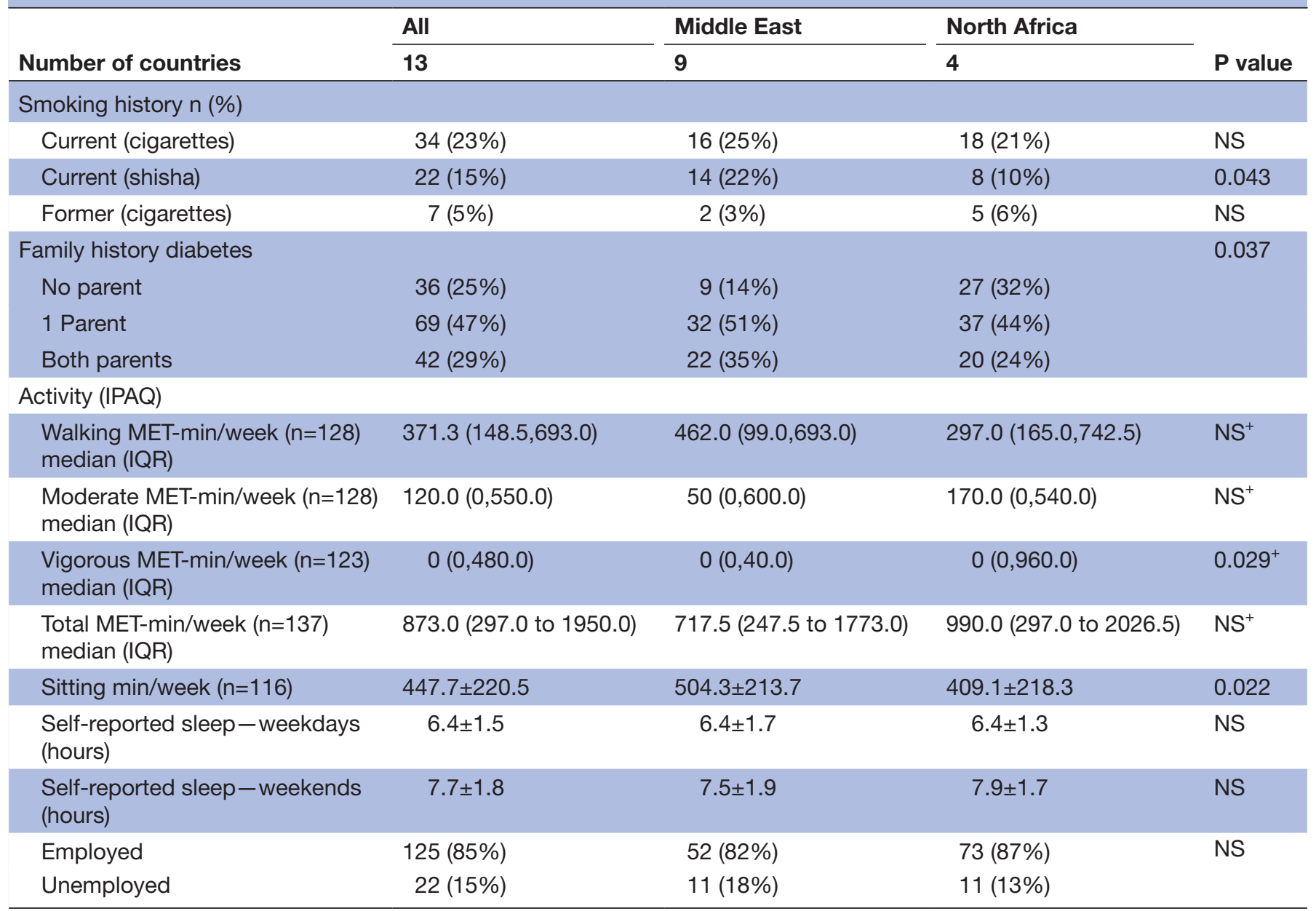

Data are percentage or mean $\pm S D$.

+, Two-sample Wilcoxon rank-sum test; BMI, body mass index; BP, blood pressure; IPAQ, international physical activity questionnaire; MET, metabolic equivalents of task; NS, non-significant ( $p>0.05)$.

by diet alone. The age, gender, anthropometrics, vital signs, diabetes duration and number of medications at baseline were not significantly different between the two regional groups (table 1). Biochemical results were also similar between the two groups (table 2). Only uric acid and lowdensity lipoprotein-cholesterol levels were significantly higher in the North Africa region study participants 369.2 $\mu \mathrm{mol} / \mathrm{L}$ (SD 90.1) versus 336.2 $\mu \mathrm{mol} / \mathrm{L}$ (SD 73.0), $\mathrm{p}=0.02$; $3.0 \mathrm{mg} / \mathrm{dL}$ (SD 0.9) versus $2.7 \mathrm{mg} / \mathrm{dL}$ (SD 0.8), $\mathrm{p}=0.038$, respectively. About one quarter (23\%) of participants were current cigarette smokers and $15 \%$ reported smoking shisha, with shisha smoking being significantly higher in the Middle East participants (22\% vs 10\%, $\mathrm{p}=0.04$ ). About three quarters $(76 \%)$ of subjects recorded having a positive family history of diabetes in at least one parent, and positive family history of diabetes was significantly higher in North Africa subjects $(\mathrm{p}=0.04)$. About one-third $(29 \%)$ reported having positive family history of T2DM in both parents.

Self-reported physical activity at baseline (measured by the international physical activity questionnaire) showed that North Africa participants did more vigorous physical activity (metabolic equivalents of task (MET)-min/week) than Middle East participants (516.6 MET-min/week (SD 838.6) vs 176.2 MET-min/week (SD 386.0), $\mathrm{p}=0.007$ ) and had less sitting time per week (409.1 min (SD 838.6) vs $504.3 \mathrm{~min}$ (SD 213.7), $\mathrm{p}=0.02$ ), indicating that North African participants were more active. Self-reported sleep showed that DIADEM-I subjects slept an average of 6.4 hours (SD 1.5) on weekdays and 7.7 hours (SD 1.8) on weekends. Table 3 shows self-reported diabetes complications and medical history. None of the participants had self-reported diabetic retinopathy or neuropathy at baseline and only $4 \%$ reported diabetic nephropathy. One-third (30\%) of study participants reported having a history of hypertension and one-third $(31 \%)$ were taking one or more antihypertensives. More than half of the participants $(52 \%)$ reported snoring at baseline. Fourteen subjects $(10 \%)$ reported a history of gout.

Table 4 uses a waist circumference median split $(112.3 \mathrm{~cm})$ to compare the characteristics of the cohort. All measures of insulin sensitivity/resistance (Quantitative Insulin Sensitivity Check Index, homeostatic model assessment of insulin resistance, homeostatic model assessment beta cell function) demonstrated significantly 
Table 2 Baseline biochemistry by region of origin

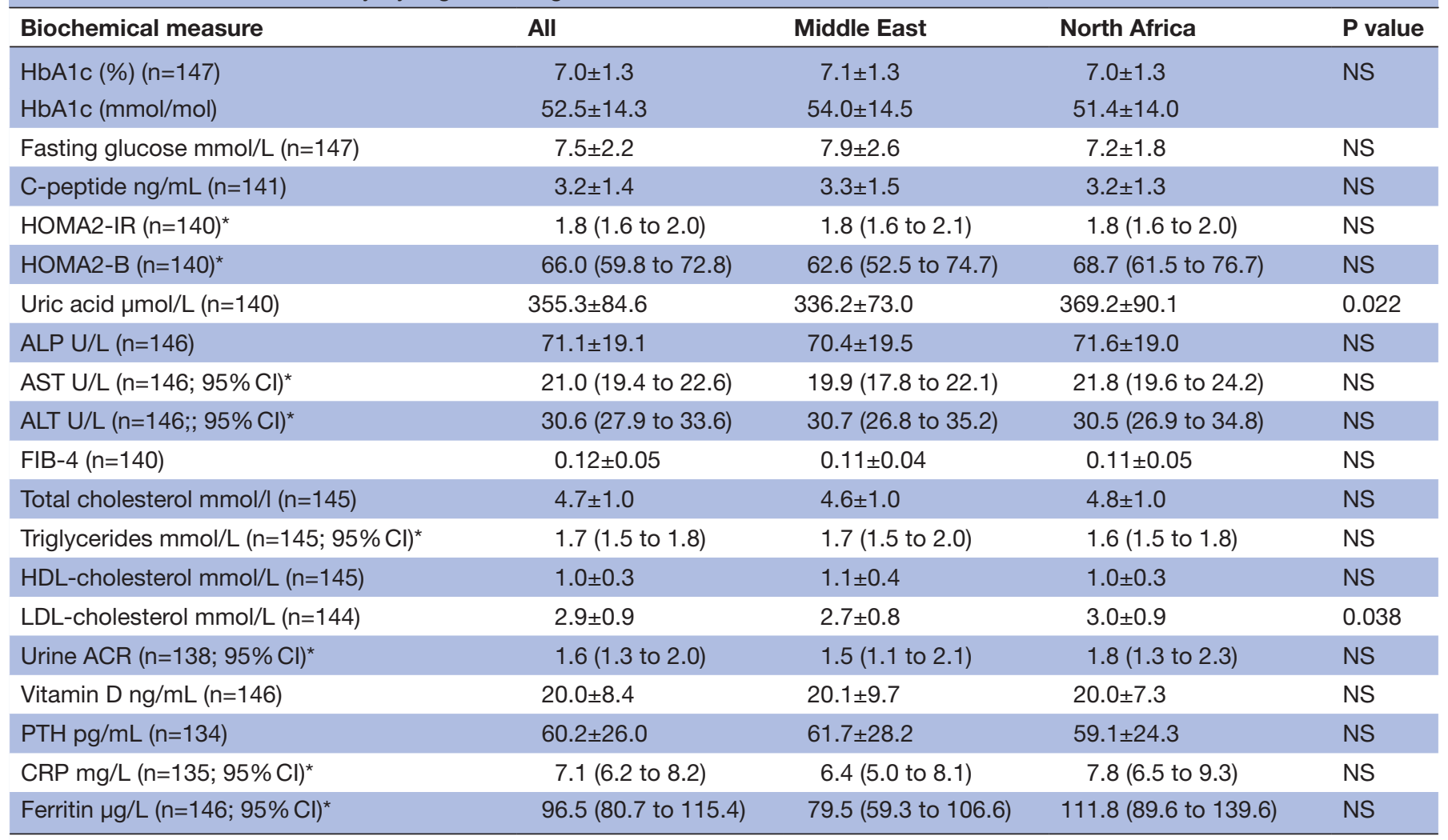

Data are mean \pm SD.

*Log-transformed for analysis - summary data are back-transformed means and $95 \% \mathrm{Cl}$.

ACR, albumin-to-creatinine ratio; ALP, alkaline phosphatase; ALT, alanine aminotransferase; AST, aspartate aminotransferase; CRP, C reactive protein; FIB-4, Fibrosis-4 Index for liver fibrosis; HDL, high-density lipoprotein; HOMA2-B, homeostatic model assessment beta cell function; HOMA2-IR, homeostatic model assessment of insulin resistance; LDL, low-density lipoprotein; NS, non-significant (p>0.05); PTH, parathyroid hormone.

greater insulin resistance with larger waist circumference. Uric acid and $\mathrm{C}$ reactive protein (CRP) levels were significantly higher in those with the larger waist circumference. Vitamin D levels were significantly higher in the smaller waist circumference group $(p=0.028)$. Quality of life (measured by Impact of Weight on Quality Of LifeLite and Euroquol 5-dimensions, EQ-5D) was significantly lower in the higher waist circumference group ( $\mathrm{p}<0.0001$ and $\mathrm{p}=0.045$, respectively).

\section{DISCUSSION}

DIADEM-I is the first randomised clinical trial in primary care in Qatar and to our knowledge, the first randomised clinical trial in the MENA region of an ILI incorporating a TDR phase using LED meal replacement products combined with physical activity and behaviour change support. The study observed a significant remission of diabetes and achievement of normoglycaemia in the lifestyle intervention participants. ${ }^{11}$

\section{Participant recruitment in Qatar}

Recruitment into clinical trials can be challenging, particularly when a trial is implemented for the first time. Our previous work suggested that the majority of participants approached for research in Qatar were willing to participate. ${ }^{15}$ The use of the electronic medical records combined with clinical discussion with potential participants ensured that the majority $(85 \%)$ of those referred to the research team were suitable for the study and were willing to participate. Of those who attended for screening, $92 \%$ agreed to participate in the study. Commonly, primary care participants are invited from a general practice data search through invitation letters by post. The uptake from a postal approach is much lower than the more personal approach employed in primary care to describe the DIADEM-I study. This approach would not have been possible without dedicated support from primary care staff. Similar to other studies, the main reason for not agreeing to participate was the time commitment required, especially for the lifestyle intervention arm which required frequent visits. ${ }^{15}$ The majority of participants, however, were in full-time employment, but were willing to commit to the intensive study visits. Ongoing qualitative analyses will examine the acceptability of the lifestyle intervention, the motivators for participation and factors associated with success in the study.

\section{Research setting}

Conducting the study in Qatar allowed access to a group of participants originating from 13 different countries, 
Table 3 Self-reported diabetes complications and medical history of participants at baseline

\begin{tabular}{|c|c|}
\hline Medical history & $\mathbf{N}(\%)$ \\
\hline $\mathrm{N}$ & 147 \\
\hline Diabetic retinopathy & 0 \\
\hline Diabetic nephropathy & $6(4 \%)$ \\
\hline Diabetic neuropathy & 0 \\
\hline \multicolumn{2}{|l|}{ Cardiovascular } \\
\hline Hypertension & $44(30 \%)$ \\
\hline Angina & 0 \\
\hline Myocardial infarction & $1(0.7 \%)$ \\
\hline Coronary stent & $1(0.7 \%)$ \\
\hline Coronary artery bypass graft & 0 \\
\hline Heart failure $\mathrm{NYHA}=1$ & $1(0.7 \%)$ \\
\hline Stroke & 0 \\
\hline Peripheral vascular disease & $1(0.7 \%)$ \\
\hline \multicolumn{2}{|l|}{ Respiratory } \\
\hline Snoring & $77(52 \%)$ \\
\hline Obstructive sleep apnoea & $6(4 \%)$ \\
\hline Asthma & $10(7 \%)$ \\
\hline Chronic obstructive pulmonary disease & 0 \\
\hline \multicolumn{2}{|l|}{ Gastrointestinal } \\
\hline Gastro-oesophageal reflux & $21(14 \%)$ \\
\hline Cholecystectomy & $10(7 \%)$ \\
\hline Non-alcoholic fatty liver disease & $5(3 \%)$ \\
\hline \multicolumn{2}{|l|}{ Musculoskeletal } \\
\hline Gout & $14(10 \%)$ \\
\hline Arthritis & $2(1 \%)$ \\
\hline
\end{tabular}

Data are frequencies and percentages.

NYHA, New York Heart Association

which supports the generalisability of DIADEM-I. The participants are also representative of the primary care younger diabetes population in Qatar which consists of about $70 \%$ men (unpublished data). The study was conducted in primary care where the majority of the early diabetes population are a community setting that provided access to gym facilities for both men and women. A major challenge faced at the beginning of the study was to find adequate clinic space to conduct the intervention, a common problem in busy primary care settings. However, the recent development of dedicated wellness centres in primary care in Qatar that provide access to gym as well as dietitians and lifestyle management physicians will ensure that the intervention can be rapidly translated into the primary care system in Qatar. An increasing trend towards emphasising wellness and greater attention to obesity and its complications across the world will facilitate reversing diabetes in primary care.

\section{Key clinical and metabolic characteristics of DIADEM-I} participants

Interestingly, DIADEM-I subjects reported an average of 6.4 hours (SD 1.5) sleep on weekdays, and 7.7 hours (SD
1.8) of sleep on weekends. Weekday sleep was below the recommendation that adults should sleep at least 7 hours per night. ${ }^{17}$ Although sleep was longer during weekend days, there is a weekly sleep deficit. Short sleep is associated with many adverse health outcomes including obesity and diabetes and this indicates that special attention should be paid to sleep in this population. ${ }^{18-20}$ Education on the importance of adequate sleep duration may aid in weight management in these individuals. Indeed, the DIADEM-I lifestyle intervention provided some sleep improvement advice. More than half of the subjects reported snoring, but only $4 \%$ reported having diagnosed obstructive sleep apnoea (OSA). It is possible that OSA may be underdiagnosed in this population and has adverse metabolic consequences. ${ }^{21}{ }^{22}$ About $10 \%$ of the DIADEM-I subjects reported a history of gout. Because of the protein content of the meal replacements, it is important to monitor uric acid levels to prevent occurrence of adverse events.

Within the DIADEM-I population, those with higher waist circumference had significantly higher fat mass, greater insulin resistance, higher uric acid levels, had a greater degree of inflammation as measured through CRP, and lower quality of life. This demonstrates the metabolic burden of excess central adiposity among young individuals with early diabetes. Although significantly lower in the higher waist circumference group, overall both groups reported having a good quality of life. Depression and anxiety measured by Hospital Anxiety and Depression Scale were low in the DIADEM-I cohort. Good quality of life and low anxiety and depression in this population are probably related to the short duration of T2DM and hence lesser disease burden.

\section{Comparison of DIADEM-I participants with those in other T2DM trials}

Onset of T2DM is increasingly occurring at a younger age, particularly in the MENA region. The incidence of T2DM in younger individuals in the MENA region was found to be one of the highest in the world compared with other regions. ${ }^{1}$ As a comparator, those recruited into the Early Activity in Diabetes (Early ACTID) study, a lifestyle intervention in UK primary care for those with early diagnosed diabetes were about 60 years old. ${ }^{23}$ Similarly, the United Kingdom Prospective Diabetes Study recruited 4209 newly diagnosed patients with T2DM and they were 52 years old, a decade older than the DIADEM-I cohort. ${ }^{24}$

Evidence from bariatric surgery supports the idea that younger age and shorter duration of diabetes are more likely to achieve diabetes remission with weight loss. ${ }^{25}$ The DIADEM-I study is novel because the intervention addressed younger individuals soon after diagnosis with the aim of improving their diabetes status and even potentially achieving remission. The DIADEM-I cohort is compared with other diabetes study populations worldwide who underwent weight loss interventions (lifestyle interventions and pharmacological treatment) in online supplemental table 1 . There are key differences between DIADEM-I and four other studies. Where all 4 trials were 
Table 4 Baseline characteristics by waist circumference median split

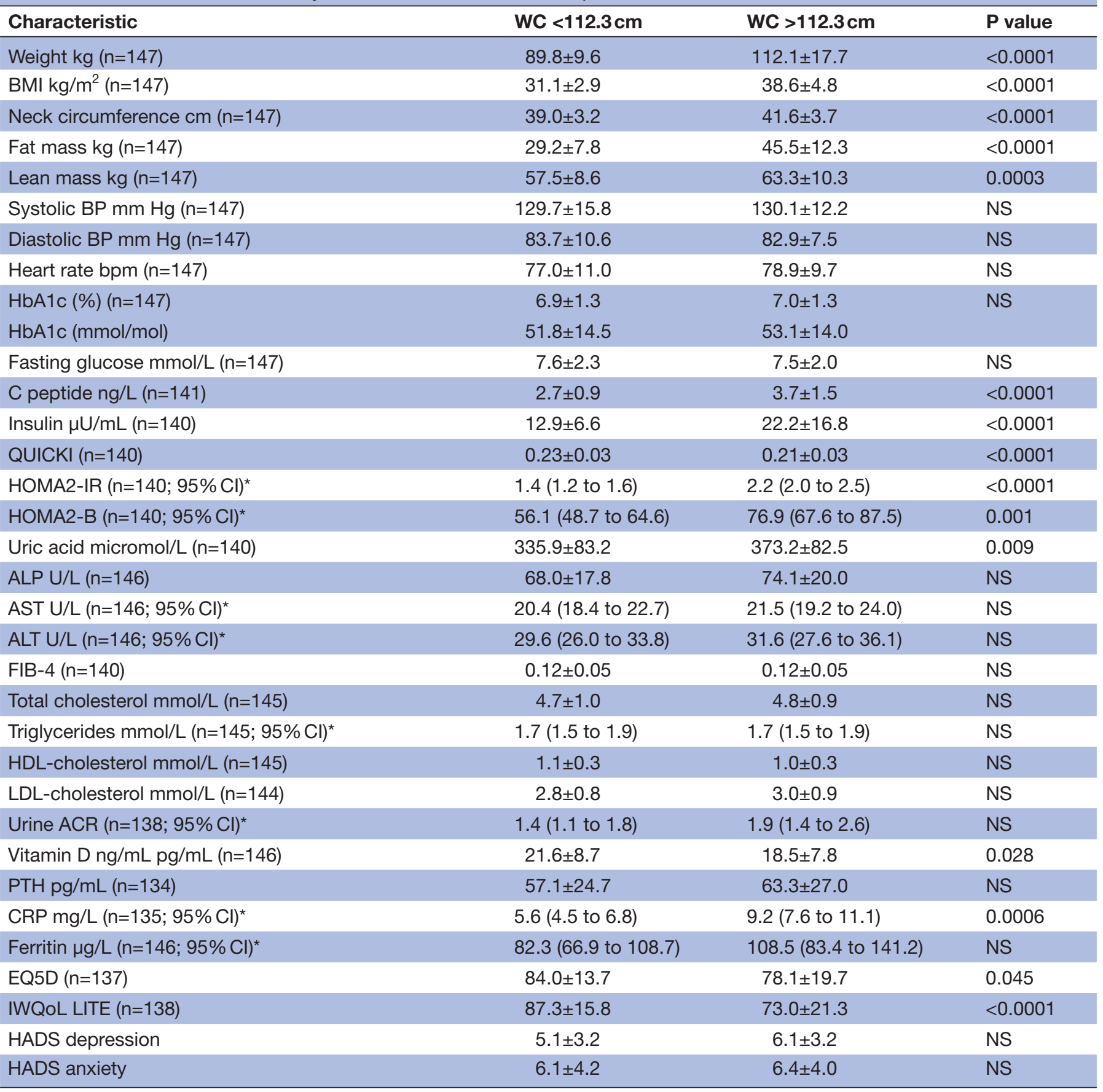

Data are mean \pm SD.

*Log-transformed for analysis - data are presented as back-transformed means and $95 \% \mathrm{Cl}$.

ACR, albumin-to-creatinine ratio; ALP, alkaline phosphatase; ALT, alanine aminotransferase; AST, aspartate aminotransferase; BMI, body mass index; CRP, C reactive protein; FIB-4, Fibrosis-4 Index for liver fibrosis; HADS, Hospital Anxiety and Depression Scale; HDL, highdensity lipoprotein; HOMA2-B, homeostatic model assessment beta cell function; HOMA2-IR, homeostatic model assessment of insulin resistance; LDL, low-density lipoprotein; IWQoL LITE, impact of weight on quality of life LITE questionnaire; NS, non-significant ( $p>0.05$ ); PTH, parathyroid hormone; QUICKI, Quantitative Insulin Sensitivity Check Index.

predominantly in white Europeans, DIADEM-I included people originating from 13 countries in the MENA region. Again, the DIADEM-I population is noticeably younger than the other cohorts (range 12-18 years younger; online supplemental figure 1). Although DIADEM-I subjects resemble the other populations in terms of most anthropometric and biochemical characteristics, it is important to note some differences. The DIADEM-I and DiRECT cohorts report a similar average BMI in their populations $\left(34.9 \mathrm{~kg} / \mathrm{m}^{2}\right.$ (SD 5.5$)$ and $34.6 \mathrm{~kg} / \mathrm{m}^{2}$ (SD 4.4), respectively). Also, HbA1c and fasting glucose levels are lower in DIADEM-I and Early ACTID subjects (online 
supplemental figure 1), indicating their shorter disease duration puts them at an advantage in terms of insulin resistance and glycaemic control and hence possible diabetes remission. Although the DIADEM-I cohort shows lower levels of comorbidities and diabetes complications (CVD, hypertension, retinopathy), probably due to the younger age of individuals and shorter diabetes duration, DIADEM-I subjects have a notably higher percentage of current smokers compared with other trials. It is also noteworthy that in DIADEM-I subjects, blood pressure targets were met, but lipid control was suboptimal. Only about $10 \%$ of DIADEM-I participants managed their diabetes by diet alone for T2DM which when compared with participants of the Early ACTID study (also with short disease duration) indicates that the regional approach seems to favour early introduction of pharmacotherapy in T2DM. ${ }^{11} 23$

\section{Study limitations}

Although the sample size is sufficient for the primary outcome of the trial to be met, it remains a relatively small focused sample. Furthermore, our findings are limited to a single study population. Study subjects originated from 13 countries. However, the study population included many long-term residents and citizens. Finally, the study population is a very selective group of patients with T2DM (younger, shorter diabetes duration) and may be used as an accurate representation of most patients with T2DM in the region.

\section{CONCLUSION}

DIADEM-I has demonstrated that it is possible to efficiently recruit participants originating from the MENA region into a randomised controlled trial incorporating an ILI. The intervention achieved significant weight loss and normoglycaemia. ${ }^{11}$ The DIADEM-I population is distinctly different from other studies mainly conducted in white European populations. Outcomes of DIADEM-I inform the care of patients with obesity and T2DM worldwide, where there is now a need to emphasise diabetes reversal through weight loss and lifestyle change as soon as T2DM is diagnosed.

\section{Author affiliations}

${ }^{1}$ Department of Medicine, Weill Cornell Medicine Qatar, Doha, Qatar

${ }^{2}$ Department of Medicine, Weill Cornell Medicine New York, New York, United States

${ }^{3}$ Qatar Metabolic Institute, Hamad Medical Corporation, Doha, Qatar

${ }^{4}$ National Obesity Treatment Center, Hamad Medical Corporation, Doha, Qatar

${ }^{5}$ Primary Health Care Corporation, Doha, Qatar

${ }^{6}$ Qatar Diabetes Association, Qatar Foundation, Doha, Qatar

${ }^{7}$ Department of Statistics and Data Science, Cornell University, Ithaca, United States

\section{Twitter Odette Chagoury @0detteChagoury and Shahrad Taheri @TaheriShahrad}

Acknowledgements The authors are grateful to the DIADEM-I participants and the WCMQ, HMC, and PHCC IRBs and WCMQ institutional DSMB. They are also grateful to colleagues at the Primary Healthcare Corporation, Doha, Qatar for participant identification and laboratory support. $\mathrm{HZ}$ is registered as a PhD candidate at King's College London (Supervisors: Dr Barbara McGowan and Professor Shahrad Taheri).

Contributors ST (principal investigator) conceived the study and designed the intervention. ST, MC, KEN, MTW and OC contributed to the study design. OC was the clinical trial lead and supported oversight of the study. HZ oversaw the day-to-day conduct of the study with support from SE and SHA. NS provided physician support. AA-H, AA-N, SA-A and ABA-S provided logistic support for study implementation. $\mathrm{HZ}, \mathrm{ST}, \mathrm{OC}$ and MTW contributed to data analysis. HZ drafted the manuscript. All authors reviewed the manuscript and approved the final version. ST is the guarantor for this work.

Funding DIADEM-I was funded by the Qatar National Research Fund through the National Priorities Research Programme grant (NPRP 8-912-3-192) awarded to ST. The statements made in this publication are solely the responsibility of the authors.

Competing interests ST has participated as an advisory board member of Novo Nordisk and reports a previous educational grant from Cambridge Weight Plan UK for an investigator initiated randomised clinical trial (ISRCTN21335883). All other authors declare no competing interests.

Patient consent for publication Not required.

Ethics approval Ethical approvals were obtained from the Weill Cornell Medicine Qatar (WCMQ; ref: 15-00071), Hamad Medical Corporation (HMC; ref: 15395_15), and Primary Health Care Corporation (PHCC; ref: PHCC/IEC/17/02/002) institutional review boards in Qatar. The study is supported by the WCMQ Institutional Data and Safety Monitoring Board.

Provenance and peer review Not commissioned; externally peer reviewed.

Data availability statement Data are available upon reasonable request. Data from the DIADEM-I study will be available from the corresponding author (staheri@ me.com) on reasonable request.

Supplemental material This content has been supplied by the author(s). It has not been vetted by BMJ Publishing Group Limited (BMJ) and may not have been peer-reviewed. Any opinions or recommendations discussed are solely those of the author(s) and are not endorsed by BMJ. BMJ disclaims all liability and responsibility arising from any reliance placed on the content. Where the content includes any translated material, BMJ does not warrant the accuracy and reliability of the translations (including but not limited to local regulations, clinical guidelines, terminology, drug names and drug dosages), and is not responsible for any error and/or omissions arising from translation and adaptation or otherwise.

Open access This is an open access article distributed in accordance with the Creative Commons Attribution Non Commercial (CC BY-NC 4.0) license, which permits others to distribute, remix, adapt, build upon this work non-commercially, and license their derivative works on different terms, provided the original work is properly cited, appropriate credit is given, any changes made indicated, and the use is non-commercial. See: http://creativecommons.org/licenses/by-nc/4.0/.

ORCID iD

Shahrad Taheri http://orcid.org/0000-0001-8314-1500

\section{REFERENCES}

1 IDF. IDF diabetes atlas. 9th ed, 2019.

2 Zawiejska A, McAleese J, Yemparala P, et al. Treatment intensification in type 2 diabetes mellitus and obesity. $\mathrm{Br} J$ Gen Pract 2013;63:182.1-182.

3 Leong WB, Taheri S. The role of bariatric surgery in the treatment of type 2 diabetes mellitus. J R Coll Physicians Edinb 2012;42:194-8.

4 Borgeraas H, Hofs $\varnothing \mathrm{D}$, Hertel JK, et al. Comparison of the effect of Roux-en-Y gastric bypass and sleeve gastrectomy on remission of type 2 diabetes: a systematic review and meta-analysis of randomized controlled trials. Obes Rev 2020;21:e13011. doi:10.1111/ obr.13011

5 Lean ME, Leslie WS, Barnes AC, et al. Primary care-led weight management for remission of type 2 diabetes (direct): an open-label, cluster-randomised trial. Lancet 2018;391:541-51.

6 Lean MEJ, Leslie WS, Barnes AC, et al. Durability of a primary care-led weight-management intervention for remission of type 2 diabetes: 2-year results of the direct open-label, cluster-randomised trial. Lancet Diabetes Endocrinol 2019;7:344-55.

7 Taylor R, Leslie WS, Barnes AC, et al. Clinical and metabolic features of the randomised controlled diabetes remission clinical trial (direct) cohort. Diabetologia 2018;61:589-98.

8 Brown A, Dornhorst A, McGowan B, et al. Low-Energy total diet replacement intervention in patients with type 2 diabetes mellitus and obesity treated with insulin: a randomized trial. BMJ Open Diabetes Res Care 2020;8:e001012.

9 Ohta M, Seki Y, Wong SK-H, et al. Bariatric/Metabolic surgery in the Asia-Pacific region: APMBSS 2018 survey. Obes Surg 2019;29:534-41. 
10 Taheri S, Chagoury O, Zaghloul $\mathrm{H}$, et al. Diabetes intervention Accentuating diet and enhancing metabolism (DIADEM-I): a randomised controlled trial to examine the impact of an intensive lifestyle intervention consisting of a low-energy diet and physical activity on body weight and metabolism in early type 2 diabetes mellitus: study protocol for a randomized controlled trial. Trials 2018;19:284.

11 Taheri S, Zaghloul $\mathrm{H}$, Chagoury O, et al. Effect of intensive lifestyle intervention on bodyweight and glycaemia in early type 2 diabetes (DIADEM-I): an open-label, parallel-group, randomised controlled trial. Lancet Diabetes Endocrinol 2020;8:477-89.

12 American Diabetes Association. Standards of medical care in diabetes--2014. Diabetes Care 2014;37 Suppl 1:S14-80.

13 Colberg SR, Sigal RJ, Fernhall B, et al. Exercise and type 2 diabetes: the American College of sports medicine and the American diabetes association: joint position statement executive summary. Diabetes Care 2010;33:2692-6.

14 Gunathilake W, Song S, Sridharan S, et al. Cardiovascular and metabolic risk profiles in young and old patients with type 2 diabetes. QJM 2010;103:881-4.

15 Tohid H, Choudhury SM, Agouba S, et al. Perceptions and attitudes to clinical research participation in Qatar. Contemp Clin Trials Commun 2017;8:241-7.

16 Nations U. Country meters. Qatar population clock. United Nations Department of Economic and Social Affairs, 2020.

17 Watson NF, Badr MS, Belenky G, et al. Recommended amount of sleep for a healthy adult: a joint consensus statement of the American Academy of sleep medicine and sleep research Society. Sleep 2015;38:843-4.
18 Arora T, Chen MZ, Cooper AR, et al. The impact of sleep debt on excess adiposity and insulin sensitivity in patients with early type 2 diabetes mellitus. J Clin Sleep Med 2016;12:673-80.

19 Taheri S, Lin L, Austin D, et al. Short sleep duration is associated with reduced leptin, elevated ghrelin, and increased body mass index. PLoS Med 2004;1:e62.

20 Taheri S, Mignot E. Sleep well and stay slim: dream or reality? Ann Intern Med 2010;153:475-6.

21 Guest JF, Panca M, Sladkevicius E, et al. Clinical outcomes and cost-effectiveness of continuous positive airway pressure to manage obstructive sleep apnea in patients with type 2 diabetes in the U.K. Diabetes Care 2014;37:1263-71.

22 Pallayova M, Banerjee D, Taheri S. Novel insights into metabolic sequelae of obstructive sleep apnoea: a link between hypoxic stress and chronic diabetes complications. Diabetes Res Clin Pract 2014; 104:197-205.

23 Andrews RC, Cooper AR, Montgomery AA, et al. Diet or diet plus physical activity versus usual care in patients with newly diagnosed type 2 diabetes: the early ACTID randomised controlled trial. Lancet 2011;378:129-39.

24 United Kingdom prospective diabetes study (UKPDS). United Kingdom prospective diabetes study (UKPDS). 13: relative efficacy of randomly allocated diet, sulphonylurea, insulin, or metformin in patients with newly diagnosed non-insulin dependent diabetes followed for three years. BMJ 1995;310:83-8.

25 Panunzi S, Carlsson L, De Gaetano A, et al. Determinants of diabetes remission and glycemic control after bariatric surgery. Diabetes Care 2016;39:166-74. 